\title{
Red Vial de Nicaragua (Optimización y Mantenimiento)
}

\author{
W. Martínez D. \\ Doctorado en Ciencias Económicas \\ FCES-LUZ, Venezuela y UNAN-Managua, Nicaragua \\ wamdmar@yahoo.com
}

(recibido/received: 12-Noviembre-2012; aceptado/accepted: 30-Abril-2013)

\section{RESUMEN}

La red vial es un patrimonio nacional, el cual es necesario: proteger, conservar, aumentar y mejorar; para apoyar el desarrollo socioeconómico de nuestro país. La actividad de mantenimiento, constituye un factor determinante que garantiza la operación satisfactoria del transporte durante la vida útil de los caminos; en sus diferentes modalidades. La ausencia de un mantenimiento preventivo y de un mantenimiento correctivo tardío, conduce a que la inversión realizada sufra una depreciación más acelerada, acortando su vida útil; obteniendo un grado de aprovechamiento menor que se traduce en una disminución de los beneficios estimados, ya que la rentabilidad no podrá ser óptima al descuidar esta función. "Con frecuencia, los costos operativos de los vehículos, excederán los costos de los departamentos viales por un factor de 10 o màs, especialmente en carreteras de gran volumen de trànsito". (Zaniewski, 1989). Por lo tanto, los costos (economía) de los usuarios deben ser tomados en cuenta al momento de proyectar la construcción de una carretera. Por tal razón el Gobierno de Nicaragua, en conjunto con las instituciones correspondientes, han asumido un rol prioritario en la ejecución y mantenimiento de la red vial. Este ensayo pretende aportar elementos cuantitativos y cualitativos, para el análisis y mejoramiento de la Red Vial.

Palabras claves: Nicaragua; infraestructura; mantenimiento; recursos; patrimonio nacional.

\begin{abstract}
The road network is a national wealth, which is necessary: to protect, to preserve, to increase and to improve; to support the socioeconomic development of our country. The activity of maintenance, it constitutes a determinant factor that guarantees the satisfactory operation of the transport during the useful life of the ways; in his different modalities. The absence of a preventive maintenance and of a corrective late maintenance, it drives to that the realized investment suffers a more intensive depreciation, shortening his useful life; obtaining a degree of minor utilization that is translated in a decrease of the estimated benefits, since the profitability will not be able to be ideal on having neglected this function. Often, vehicle operating costs, exceed the costs of road departments by a factor of 10 or more, especially in high-volume road traffic. (Zaniewski, 1989). Therefore, The cost (economics) of users should be taken into account when designing the construction of a road. For this reason, the Government of Nicaragua, in conjuction with the relevant institutions have assumed a primary role in the implementation and maintenance of the road network. This test tries to contribute qualitative and quantitative elements, for the analysis and improvement of the Road Network.
\end{abstract}

Keywords: Nicaragua; infrastructure; maintenance; resources; national wealth.

\footnotetext{
*Autor para la correspondencia
} 


\section{W. Martínez D}

\section{INTRODUCCIÓN}

\section{Antecedentes}

"Un pavimento puede definirse como la superestructura de la obra vial que hace posible la circulación expedita de los vehículos, con la seguridad, comodidad y economía requerida por el usuario y provistos por el proyecto". (Salazar Rodríguez y otros. 2002). En el año de 1940, época en la cual fue creado el Departamento de Carreteras del Ministerio de Obras Públicas (MOOP), hoy Ministerio de Transporte e Infraestructura (MTI), en Nicaragua existían 201 kilómetros de carreteras de los cuales solamente 52 kilómetros eran pavimentados. Este departamento se constituyó como el organismo oficial responsable a nivel nacional de la construcción y mantenimiento de las carreteras del país y sus obras complementarias; habiéndose construido todas las carreteras por administración directa, exceptuando algunos contratos para la construcción de la carretera Interamericana y la carretera al Rama, que fueron adjudicados al BUREAU OF PUBLIC ROADS (Oficina de Caminos Públicos), mediante convenio de ayuda suscrito entre los gobiernos de Nicaragua y los Estados Unidos de América, que suministró la supervisión y asesoramiento técnico a lo largo del proceso de construcción de las carreteras.

En 1951, en el Departamento de Carreteras se dieron un sinnúmero de innovaciones técnicas, que acompañada de una política clara dirigida al desarrollo de la infraestructura vial, conllevó al desarrollo de vastas regiones del país, ligadas a la importancia económica de estas. Estos cambios permitieron reducciones importantes en el tiempo que se invertía en los traslados, así como en los riesgos para las personas y mercancías, lo cual se tradujo en disminución significativa en los costos.

A partir de 1955, los financiamientos aumentaron y las inversiones ajustadas a presupuestos particulares, trajeron como resultado un nuevo empuje a la expansión de la red vial. A esa fecha existían en Nicaragua 3,687 kilómetros de carreteras, contando con 285 kilómetros de carreteras pavimentadas. Ya en 1965, se tenían 6,475 kilómetros, de los cuales 811 eran pavimentados.

El terremoto de 1972, en alguna medida, incidió directamente en el primer freno del desarrollo de la Red Vial, sin duda esto fue solamente un síntoma del gran efecto que el desastre natural tuvo en la economía del país, misma que se logra superar en cierta medida, de manera inmediata; gracias al gran apoyo internacional ofrecido tanto en ayuda humanitaria como económica.

El segundo y más dramático momento fue después de 1977, año en que prácticamente las inversiones a la red vial, se mantienen congeladas durante cinco años consecutivos, para terminar en 1984 con un descenso lamentable tanto en infraestructura como en deterioro de la red, producto de ciertas condiciones especiales, que en los periodos de guerra, se generan en un país, y de la cual Nicaragua era objeto.

Las metodologías de diseños de espesores de pavimentos en Estados Unidos, tienen su antecedente fundamental en los trabajos a escala real, realizados en el tramo de ensayos AASHO Road Test de 1960, en Illinois, Chicago. Los resultados de los estudios fueron publicados en la primer "Guía AASHO para Diseño de Pavimentos Rígidos y Flexibles. Así sucesivamente se han hecho varias revisiones, hasta llegar a la "Guía para el Diseño de Estructuras de Pavimento", conocida en inglés como la "AASHTO Guide for the Design of Pavement Structures 1993". (Soluciones de Pavimentos en Nicaragua: Comparación Técnica y Económica, I.C.C. Ingenieros Consultores Centroamericanos, S.A. e INCYC, Instituto Nicaragüense del Cemento y del Concreto, Nicaragua, pp. 5).

Además de ello, se tienen como referencia las investigaciones y conocimientos del desempeño de los pavimentos, realizados por Strategic Highway Research Program (SHRP). Algunos otros trabajos e investigaciones, relacionados al tema y puestos en práctica, acorde a las posibilidades de cada país; son los realizados por: Midwestern Pavement Preservation Partnership, Northeastern Pavement Preservation Partnership (NEPPP-2004), Southeast Pavement Preservation Partnership (SEPPP). Así como las normas de la ASTM, AASHTO y SAE.

\section{Planteamiento del problema}

En Nicaragua, el mantenimiento de caminos como actividad fundamental para la conservación de la red vial nacional, ha estado desde sus inicios bajo la dirección y administración del Ministerio de Transporte e Infraestructura (MTI). Desde mediados de los años cincuenta hasta prácticamente 1986, la estructura administrativa que estaba a cargo del mantenimiento de caminos, era un ente presupuestado del Estado, que realizaba labores de mantenimiento como una obligación, en toda la red vial. En este periodo, durante la década de los setentas, el Departamento de 
Carreteras, se transforma en la Dirección General de Caminos; que contaba con ocho zonas de mantenimiento, las que existían desde mediados de los años setenta.

En Nicaragua, antes del año de 1996, el diseño de pavimentos siguiendo la metodología AASHTO, de multicapas elásticas, fue relativamente inexistente. Hasta finales de los años ochenta, en Nicaragua, las metodologías de diseño de pavimentos conocidas, eran totalmente empíricas. Dos metodologías bastante utilizadas por ingenieros de suelos y pavimentos fueron: la conocida como "Murillo López de Souza", que se basaba en características de los suelos (Índice de Grupo) y categorías de tráfico en base a "vehículos comerciales"; y la primera versión de la Guía de Diseño para países tropicales y sub-tropicales, publicada por el Transportation Research Laboratory (TRL, Gran Bretaña) e introducida en el país por especialistas de origen danés (a atraves de la colaboración de la agencia Danida).

A partir de 1979, las zonas de mantenimiento se transforman en delegaciones regionales. En 1986, estas delegaciones regionales que continuaron realizando las labores de mantenimiento como instancias presupuestadas, sufren un cambio, se transforman en Empresas Regionales de la Construcción, y, por primera vez se realizan los trabajos de mantenimiento bajo la modalidad de contratos de obras. Debe recalcarse, que estas empresas regionales, además del mantenimiento, tenían bajo su responsabilidad la ejecución de construcción de caminos en sus diferentes modalidades. Dichas empresas están siempre adscritas al MTI y son conocidas como Corporación de Empresas Regionales de la Construcción (COERCO), las cuales se encuentran a la fecha en proceso de reordenamiento.

A partir del año 2000, a través de la Ley no. 355, ley creadora del Fondo de Mantenimiento Vial (FOMAV), y su reforma Ley no. 572, de acuerdo a la ley no. 290 (Ley de organización, competencia y procedimientos del poder Ejecutivo); que se destina un fondo para la gestión del mantenimiento de la red vial. Se propuso ante la Asamblea Nacional el cobro de USD 0.20 por cada galón de combustible que se importe. Dicho organismo parlamentario decidió que no se aplicara dicho cobro y que la asignación al FOMAV se diera a través de una partida presupuestaria. Durante los años 2000 al 2002, el Ministerio de Hacienda y Crédito Público (MHCP), no realizó ningún desembolso, ni transferencia presupuestaria al respecto.
Fue a mediados del año 2003, que el Banco Interamericano de Desarrollo (BID) y el Banco Mundial (BM), disponen de una partida presupuestaria de USD 7.0 millones, como fondo semilla para la gestión del mantenimiento. Al final dicho desembolso fue de USD 5.8 millones. Dicha gestión de mantenimiento inició con 150 a 200 kilómetros, de toda la red vial de Nicaragua, lo cual, fue un inicio. En diciembre del 2005, con la aparición y gestión de obras de parte de la Cuenta Reto del Milenio (USA), condiciona al gobierno de la República de Nicaragua, la asignación de recursos al FOMAV; los cuales son aprobados en sesión ordinaria de la Asamblea Nacional, por el orden de USD 0.16 por cada galón de combustible importado.

A pesar de ello, se inició en el año 2006, con USD 0.06 y paulatinamente se llega al año 2010 con USD 0.16, a través de la importación del petróleo. El MTI firma anualmente un convenio de trabajo con el FOMAV. De lo recaudado en concepto de este rubro, el $80 \%$ es para el FOMAV y el $20 \%$ restante, se transfiere a los municipios de Nicaragua. En el tiempo presente, dicha recaudación asciende aproximadamente a USD 32.0 millones, de los cuales:

\section{$80 \%$ FOMAV USD 25.6 millones \\ $20 \%$ Municipios USD 6.4 millones}

De lo asignado al FOMAV, USD 1.6 millones corresponden a gastos operativos y USD 24.0 millones al mantenimiento de la Red Vial.

Los problemas en la Red Vial de Nicaragua, responden en líneas generales a tres tipos de causas:

a) Estructurales: Un buen porcentaje de la Red Vial, tiene más de 30 años de haber sido construida, lo que evidencia el vencimiento de su vida útil.

b) Naturales: Otra causa fundamental para el deterioro de la Red Vial, es el exceso de lluvias y el tipo de suelos.

c) Institucionales o de gestión: Estas están referidas a lo inadecuado de la administración de las carreteras, por parte de las autoridades del sector y otras previstas en las normas, especificaciones técnicas, términos de referencia; así como, la falta de actualización de las mismas. Entre estas, podemos mencionar: a) fallas en el control del uso de las vías (el sobrepeso es un ejemplo claro de esto), y b) las autoridades (sectoriales o globalistas), no consideran un monto de recursos adecuados, para el mantenimiento de dicha infraestructura. 
El objetivo de este artículo es formular lineamientos y estrategias esenciales en la Optimización de recursos para el fortalecimiento de la Red Vial, estableciendo parámetros de referencia para tal fin. Así como, determinar los factores fundamentales que inciden directamente en la vida útil de la Red Vial y analizar alternativas para reducir los costos de las labores de rehabilitación y mantenimiento. De esta forma se podrán reducir los costos de producción de los usuarios, dotando a la población de una mejor infraestructura vial y mejorando el desarrollo integrado económico, a nivel nacional.

Al incorporar en el diseño y administración vial, conocimientos sobre la evaluación del impacto ambiental y la vulnerabilidad de la infraestructura vial; la inversión realizada será de mayor duración y a un costo a largo plazo menor.

\section{Importancia y justificación}

El presente estudio es de vital importancia para el país, dado que la red vial, es un patrimonio nacional. La actividad de rehabilitación y de mantenimiento de caminos, son vitales para el desarrollo socioeconómico de un país. El enfoque de este estudio y su aportación al conocimiento, tiene un enfoque dinámico para futuras investigaciones, tanto a nivel de pregrado, postgrado, maestría y doctorado; tanto por las variables endógenas y exógenas que se presentan al respecto, como por el aporte científico para tal fin.

En Nicaragua, dicha actividad se ha visto afectada por varios factores, entre los que sobresalen:

- Falta de Recursos Financieros: Actualmente, el FOMAV ejecuta un $82.8 \%$ (promedio) del Plan Operativo programado (FOMAV, 2009. Memoria de Labores, pp.20), teniendo como resultado en los últimos diez años un arrastre de ejecución de obras, que se acumulan y vuelven insuficientes los esfuerzos de gestión del mantenimiento y recursos financieros asignados, causando entre otros un deterioro mayor en la red vial y afectaciones socioeconómicas, que van en detrimento del desarrollo integral del país, sobre todo por afectaciones directas de los desastres naturales. En el caso del Ministerio de Transporte e Infraestructura, ver Ejecución Física Financiera a Octubre 2012. (Tabla7: Avance Físico en Km.)(Tabla 8: Avance Financiero-millones de C\$). (Fuente: Seguimiento PIP, MTI).
Se deberían de ejecutar anualmente $5,000 \mathrm{~km}$ y se ejecutan $4,130 \mathrm{~km}$; creando un déficit de ejecución de obras de $870 \mathrm{~km}$ que representan un $17.2 \%$ de lo programado nominal. Para mejorar dicha situación, es necesario contar con mayor voluntad política y asignación de presupuesto en dicho rubro, así como la agilización de las licitaciones correspondientes en cada caso.

- La apertura económica del país en 1,990 significó un crecimiento en el parque automotor. Entre 1985 y 1995, según estadísticas aportadas por la Dirección General de Aduanas (DGA), el crecimiento fue de 118,335 a 248,642 , con una tasa de crecimiento anual del $7.7 \%$; aumentando también con ello, la congestión vehicular y la tasa de accidentes de tránsito. La solución de esta situación pasa por una adecuada formulación y evaluación de proyectos de carreteras, para que, con esta base, se puedan solicitar y tramitar el financiamiento requerido; el que de todas maneras, tiene un perfil de mediano y largo plazo.

- A la fecha se han hecho esfuerzos varios, para normalizar los procesos de revisión de estudios y diseños viales, así como en la ejecución y supervisión de los mismos, siendo los más recientes, los Manuales realizados por la firma de Ingenieros Consultores, Corea y Asociados (CORASCO) y financiados con el apoyo del gobierno del Reino de Dinamarca, a través del programa de apoyo al sector transporte PAST DANIDA II Fase, que está aportando al fortalecimiento de la Oficina de Inventario Vial (MTI); reflejado este en la realización de una consultoría para el desarrollo de un sistema de inventario vial bajo la plataforma de SQL server 2005, que permitirá en fechas próximas actualizar, administrar, realizar consultas a la base de datos de la red vial, vinculadas a la cartografía digital en ArcMap en el menor tiempo posible. Ello implicará necesariamente el estudio de los mismos e iniciar su pronta aplicación en proyectos de infraestructura y transporte.

- Esta herramienta nos debe ser de mucha utilidad, tanto para los equipos de profesionales en el área de planificación, como para las áreas que administran proyectos de construcciones viales y profesionales independientes, puesto que todos estamos involucrados en diversos momentos en el proceso de elaboración y revisión de estudios y diseños. 
Es necesario además, que iniciemos una etapa de monitoreo, para llevar registros acerca de los resultados de la aplicabilidad de estos manuales, de forma que en el futuro se puedan hacer las adecuaciones y actualizaciones que se consideren necesarias, previas evaluaciones expost.

\section{METODOLOGÍA}

El presente artículo, está orientado a la Red vial de Centroamérica como elemento exógeno y a la Red Vial de Nicaragua como elemento endógeno. El mismo trata de analizar y exponer la situación actual de la red vial. Se propone además, definir las líneas de acción a tomar, para el mejoramiento y rehabilitación de la red vial nacional, así como el aprovechamiento de los recursos con que se cuentan y plantear mejoras técnicas y administrativas al respecto.

El periodo objeto de estudio, está comprendido del año 2000 al año 2010, (y algunas pequeñas observaciones al 2012), dado que en el mismo periodo se diò una disminución en la extensión de la Red Vial: año 2001 ($1.68 \%)$ y año $2002(-3.23 \%)$, en relación al total del año 2000. Así mismo, el crecimiento de la Red Vial en el mismo periodo objeto de estudio fue del $16.18 \%$, en contraste con el periodo 1990-2000, cuyo crecimiento fue del orden del $24.49 \%$. Se hará un análisis cuantitativo y cualitativo, basado en fuentes primarias, secundarias y estadísticas de lo acontecido y ejecutado, en dicho periodo. Siendo objetivo y enfocado en la realidad nacional, con recomendaciones técnicas $\mathrm{y}$ administrativas al respecto.

El contenido de este artículo se sustentará en información obtenida a través de fuentes primarias, memorias institucionales, documentos estadísticos al respecto, políticas de gobierno durante el periodo de investigación, así como un análisis de los aciertos y desaciertos ejecutados, en proceso y mejoras a corto, mediano y largo plazo.

Para la elaboración del estudio, se utilizan como componentes básicos de la metodología a aplicar, lo siguiente:

a) Recopilación, estudio y análisis de documentación bibliográfica existente relativo a infraestructura vial de Nicaragua. En líneas generales, planificación vial, planes de inversiones, situación actual, fuentes de financiamiento, otros. b) Visitas a las instituciones y organizaciones vinculadas con el aspecto de infraestructura del país: Ministerio de Transporte e Infraestructura (MTI), Instituto de Desarrollo Rural (IDR), Instituto Nicaragüense de Fomento Municipal (INIFOM), Cámara Nicaragüense de la Construcción (CNC), Empresas Consultoras, Fondo de Mantenimiento Vial (FOMAV), FISE (Fondo de Inversión Social de Emergencia), otros.

c) Análisis de las pocas estadísticas disponibles.

d) Conformación de un directorio de datos, que refleje toda la información posible de las instituciones, organismos y organizaciones; que se encuentren relacionadas con el tema de infraestructura vial en el país.

e) Elaborar documento que recoja los indicadores estadísticos objeto de este estudio y vinculados al sector infraestructura vial. De forma tal que podamos identificar el costo por kilómetro de carreteras: pavimentadas, adoquinadas, rehabilitada o mantenida.

f) Definir la infraestructura prioritaria en la red vial (carretera panamericana y zona norte), integrando la caracterización y el criterio vial de lo más recomendable técnicamente, o lo que, las instituciones vinculadas esperan efectuar.

g) Comentarios.

\section{RESULTADOS Y DISCUSIÓN}

Nicaragua se ha dado cuenta que en materia de infraestructura vial tiene, literalmente, mucho camino por recorrer. La Red Vial, apenas aumentó en los últimos 30 años, pasando en 1980 de 18,137 km a tener 22,111 en 2010 , lo que significa un progreso de 3,974 $\mathrm{km}$ (21.9\%). La Tabla 1, presenta el Crecimiento Histórico de la Red Vial (1940-2010).

Durante el mismo periodo, las vías terrestres pavimentadas, adoquinadas y revestidas, aumentaron en $2.024 \mathrm{~km}$. Es necesario destacar que el mayor crecimiento de la Red Vial nacional, se localiza en la Zona Central y Pacífica del país (Anuario de Aforos de Tráfico, MTI, Dirección General de Planificación, 2008, pp.10), lo cual ha incidido en el crecimiento económico de los municipios localizados en dichas zonas. Por otro lado, se reconoce que debe dársele mayor gestión y recursos, al mantenimiento de la red vial, en las zonas Norte y Atlántico del país. La Tabla 2, nos muestra el 


\section{W. Martínez D}

Incremento en el inventario de la Red Vial durante el año 2010.

Existen Organismos u Organizaciones Internacionales, así como Gobiernos, que apoyan el desarrollo de la red vial nacional, ya sea por medio de préstamos o donaciones, estos son: Organización de Estados Americanos (OEA), Banco Mundial (BM) a través de la Asociación Internacional de Fomento (AIF), Banco Interamericano de Desarrollo (BID), Banco Centroamericano de Integración Económica (BCIE), Unión Europea (UE), Fondo Nórdico para el Desarrollo (FND), otros. Así como, los Gobiernos de : Japón, España, Dinamarca por medio de DANIDA y PASTDANIDA, Holanda, Alemania, otros cooperantes internacionales. La tabla 3 y la Fig. 1, nos muestran el crecimiento de la Red Vial de Nicaragua en el periodo 2007-2010.

Del total de la Red Vial nacional, el $37.9799 \%$ de caminos, se han definido (MTI y FOMAV), como Red Vial Básica (Mantenible). El Gobierno actual está haciendo grandes avances en ampliar dicha cobertura.
Tipos de clasificaciones usadas en Nicaragua:

- Por el tipo de Construcción: Carreteras Pavimentadas, Caminos Revestidos, Caminos de Todo Tiempo, Caminos de Estación Seca.

- Administrativa: Nacionales de Primera Clase; Nacionales de Segunda Clase; Departamentales de Primera Clase; Departamentales de Segunda Clase; Caminos Vecinales.

- Por su función: Arterias; Caminos Vecinales y Calles; Colectoras.

Esta clasificación fue elaborada en 1976, por la firma consultora Wilbur Smith Asociados y Cisneros y Conrado. Los criterios que las sustentan tienen su base en las recomendaciones y normas como los Congresos Panamericanos de Carreteras, auspiciados por la Organización de Estados Americanos (OEA) y del cual Nicaragua es miembro. En consecuencia, técnicamente, es la clasificación que mejor se adecúa a las condiciones reales del sistema vial nacional. 
Tabla 1. Crecimiento histórico de la red vial

\section{$m \mathbf{k i}^{\mathbf{1}}$ \\ Red Vial - 2010 \\ DIVISION GENERAL DE PLANIFICACION}

Administración Vial / Inventario de la Infraestructura de Transporte

\begin{tabular}{|c|c|c|c|c|c|}
\hline \multicolumn{6}{|c|}{ CRECIMIENTO HISTORICO DE LA RED VIAL } \\
\hline ANOS & PAY. & REV. & T.T. & E. S. & TOTAL \\
\hline 1940 & 52 & & 24 & 125 & 201 \\
\hline 1941 & 70 & & 52 & 176 & 298 \\
\hline 1942 & 94 & & 60 & 142 & 296 \\
\hline 1943 & 150 & & 120 & 162 & 432 \\
\hline 1944 & 180 & & 200 & 72 & 452 \\
\hline 1945 & 206 & & 232 & 20 & 458 \\
\hline 1946 & 206 & & 266 & 45 & 517 \\
\hline 1947 & 205 & & 280 & 30 & 515 \\
\hline 1948 & 205 & & 292 & 20 & 517 \\
\hline 1949 & 205 & & 295 & 100 & 600 \\
\hline 1950 & 235 & & 255 & 100 & 590 \\
\hline 1951 & 235 & & 255 & 100 & 590 \\
\hline 1952 & 195 & & 420 & 2,100 & 2,715 \\
\hline 1953 & 210 & & 470 & 2,400 & 3,080 \\
\hline 1954 & 250 & & 560 & 2,500 & 3,310 \\
\hline 1955 & 280 & & 707 & 2,700 & 3,687 \\
\hline 1956 & 300 & & 787 & 3,000 & 4,087 \\
\hline 1957 & 362 & & 804 & 3,200 & 4.466 \\
\hline 1958 & 515 & & 1,164 & 3,400 & 5,079 \\
\hline 1959 & 548 & & 1,170 & 3,400 & 5,118 \\
\hline 1960 & 669 & & 1,868 & 3,600 & 6,137 \\
\hline 1961 & 747 & & 1,790 & 3,600 & 6.137 \\
\hline 1962 & 761 & & 1,790 & 3,600 & 6,151 \\
\hline 1963 & 802 & & 1,790 & 3,600 & 6,192 \\
\hline 1964 & 800 & & 1,825 & 3,575 & 6,200 \\
\hline 1965 & 811 & & 2,114 & 3,550 & 6,475 \\
\hline 1966 & 878 & & 2,261 & 3,550 & 6,689 \\
\hline 1967 & 1,086 & 955 & 2,757 & 5,154 & 9,952 \\
\hline 1968 & 1,123 & 1,015 & 2,901 & 5,136 & 10,175 \\
\hline 1969 & 1,141 & 1,032 & 3,387 & 5,641 & 11,201 \\
\hline 1970 & 1,235 & 1,130 & 3,685 & 6,927 & 12,977 \\
\hline 1971 & 1,283 & 1,134 & 3,965 & 6,943 & 13,325 \\
\hline 1972 & 1.336 & 1,121 & 4,054 & 6,391 & 12,902 \\
\hline 1973 & 1336 & 1121 & 4054 & 6391 & 12902 \\
\hline 1974 & 1442 & 1189 & 5067 & 8034 & 15732 \\
\hline 1975 & 1505 & 1607 & 4831 & 9210 & 16953 \\
\hline 1976 & 1546 & 1751 & 5002 & 9626 & 17925 \\
\hline 1977 & 1560 & 2549 & 4655 & 9373 & 18137 \\
\hline 1978 & 1,560 & 2,549 & 4,655 & 9,373 & 18,137 \\
\hline 1979 & 1,560 & 2,549 & 4,655 & 9,373 & 18,137 \\
\hline 1980 & 1.560 & 2,549 & 4,655 & 9,373 & 18,137 \\
\hline 1981 & 1,560 & 2,549 & 4,655 & 9,373 & 18,137 \\
\hline 1982 & 1.549 & 2,206 & 5,033 & 5,629 & 14,417 \\
\hline 1983 & 1.549 & 2,206 & 5,033 & 5,629 & 14.417 \\
\hline 1984 & 1,565 & 2,383 & 5,019 & 5,608 & 14,575 \\
\hline 1985 & 1.569 & 2,758 & 5,064 & 5,606 & 14,997 \\
\hline 1986 & 1.569 & 2,758 & 5,064 & 5,606 & 14,997 \\
\hline 1987 & 1,569 & 2,758 & 5,064 & 5,606 & 14,997 \\
\hline 1988 & 1.598 & 2,802 & 5,171 & 5,716 & 15,287 \\
\hline 1989 & 1,598 & 2,802 & 5,171 & 5,716 & 15,287 \\
\hline 1990 & 1.598 & 2.802 & 5,171 & 5,716 & 15,287 \\
\hline
\end{tabular}


Rod Vial -2010

DIVISIÓN GENERAL DE PLALIIFICACIÓN Administración Vial / Inventario de la Infraestructura de Transporte

\begin{tabular}{|c|c|c|c|c|c|}
\hline \multicolumn{6}{|c|}{ CRECIMIENTO HISTORICO DE LA RED VIAL } \\
\hline ANOS & PAV. & REV. & T. T. & E.S. & TOTAI \\
\hline 1991 & 1,598 & 2,802 & 5,171 & 5,716 & 15,287 \\
\hline 1992 & 1,598 & 2,802 & 5,171 & 5,716 & 15,287 \\
\hline 1993 & 1,598 & 2,802 & 5,171 & 5,716 & 15,287 \\
\hline 1994 & 1,717 & 2,150 & 5,002 & 8,277 & 17,146 \\
\hline 1995 & 1,717 & 2,150 & 5,002 & 8,277 & 17,146 \\
\hline 1996 & 1,746 & 2,135 & 5,716 & 8,414 & 18,011 \\
\hline 1997 & 1,749 & 2,149 & 6,114 & 8,435 & 18,447 \\
\hline 1998 & 1,835 & 2,381 & 6,299 & 8,431 & 18,946 \\
\hline 1999 & 1,948 & 2,737 & 6,208 & 7,851 & 18,744 \\
\hline 2000 & 2,095 & 2,885 & 6,058 & 7,994 & 19,032 \\
\hline 2001 & 2,126 & 2,806 & 6,165 & 7,615 & 18,712 \\
\hline 2002 & 2,182 & 2,764 & 5,981 & 7,491 & 18,418 \\
\hline 2003 & 2,206 & 3,073 & 5,950 & 7,436 & 18,665 \\
\hline 2004 & 2,299 & 3,363 & 6,278 & 7,096 & 19,036 \\
\hline 2005 & 2,423 & 3,275 & 6,386 & 7,054 & 19,138 \\
\hline 2006 & 2,439 & 3,403 & 6,662 & 7.137 & 19,641 \\
\hline 2007 & 2,440 & 3,539 & 7,060 & 7,294 & 20,333 \\
\hline 2008 & 2,503 & 3,630 & 8,053 & 7,256 & 21,442 \\
\hline 2009 & 2,553 & 3,905 & 8,335 & 7,183 & 21,975 \\
\hline 2010 & 2,814 & 3.730 & 8,388 & 7,178 & 22,111 \\
\hline
\end{tabular}

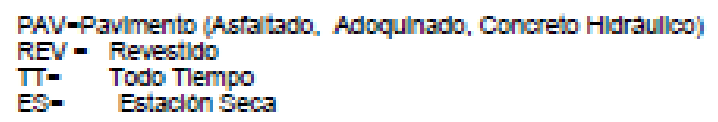

De conformidad al inventario realizado en el 2010, la Red Vial nacional registra 22,111.052 km, de los cuales:

Tabla 2. Incremento en el inventario de la red vial 2010

\begin{tabular}{|l|r|r|}
\hline & \multicolumn{1}{|l|}{ Km } & \multicolumn{1}{l|}{} \\
\hline & & \\
\hline ADOQUINADOS & $\mathbf{6 5 9 . 6 8 6}$ & $\mathbf{2 . 9 8}$ \\
\hline ASFALTADOS & $\mathbf{2 , 1 4 0 . 9 8}$ & $\mathbf{9 . 6 8}$ \\
\hline REVESTIDOS & $\mathbf{3 , 7 3 0 . 3 3}$ & $\mathbf{1 6 . 8 7}$ \\
\hline TODO TIEMPO & $\mathbf{8 , 3 8 8 . 4 5}$ & $\mathbf{3 7 . 9 4}$ \\
\hline ESTACION SECA & $\mathbf{7 , 1 7 8 . 0 7}$ & $\mathbf{3 2 . 4 6}$ \\
\hline CONCRETO & $\mathbf{1 3 . 5 4}$ & $\mathbf{0 . 0 6 1}$ \\
HIDRAULICO & & \\
\hline
\end{tabular}

(Elaboración con base a datos sistematizados de la División General de Planificación-MTI) 
Tabla 3. Crecimiento de la red vial 2007-2010

\begin{tabular}{|c|c|c|c|c|c|c|c|}
\hline AÑ̃S & ADOQUINADO & ASFALTADO & REVESTIDO & TODO & ESTACION & CONCRETO & TOTAL \\
\hline & & & & TIEMPO & SECA & \multicolumn{2}{|l|}{ HIDRAULICO } \\
\hline 2007 & 395 & 2045 & 3539 & 7060 & 7294 & - & 20333 \\
\hline 2008 & 435 & 2067 & 3630 & 8053 & 7256 & - & 21442 \\
\hline 2009 & 475 & 2070 & 3905 & 8335 & 7183 & 8 & 21975 \\
\hline 2010 & 660 & 2141 & 3730 & 8388 & 7178 & 14 & 22111 \\
\hline $2010 \%$ & 3 & 10 & 17 & 38 & 32 & $\mathbf{0}$ & 100 \\
\hline Crecimiento & & & & & & & \\
\hline km (2009-2010) & 185 & 71 & 175 & 53 & 5 & 6 & 136 \\
\hline
\end{tabular}

(Elaboración con base a datos sistematizados de la División General de Planificación-MTI)

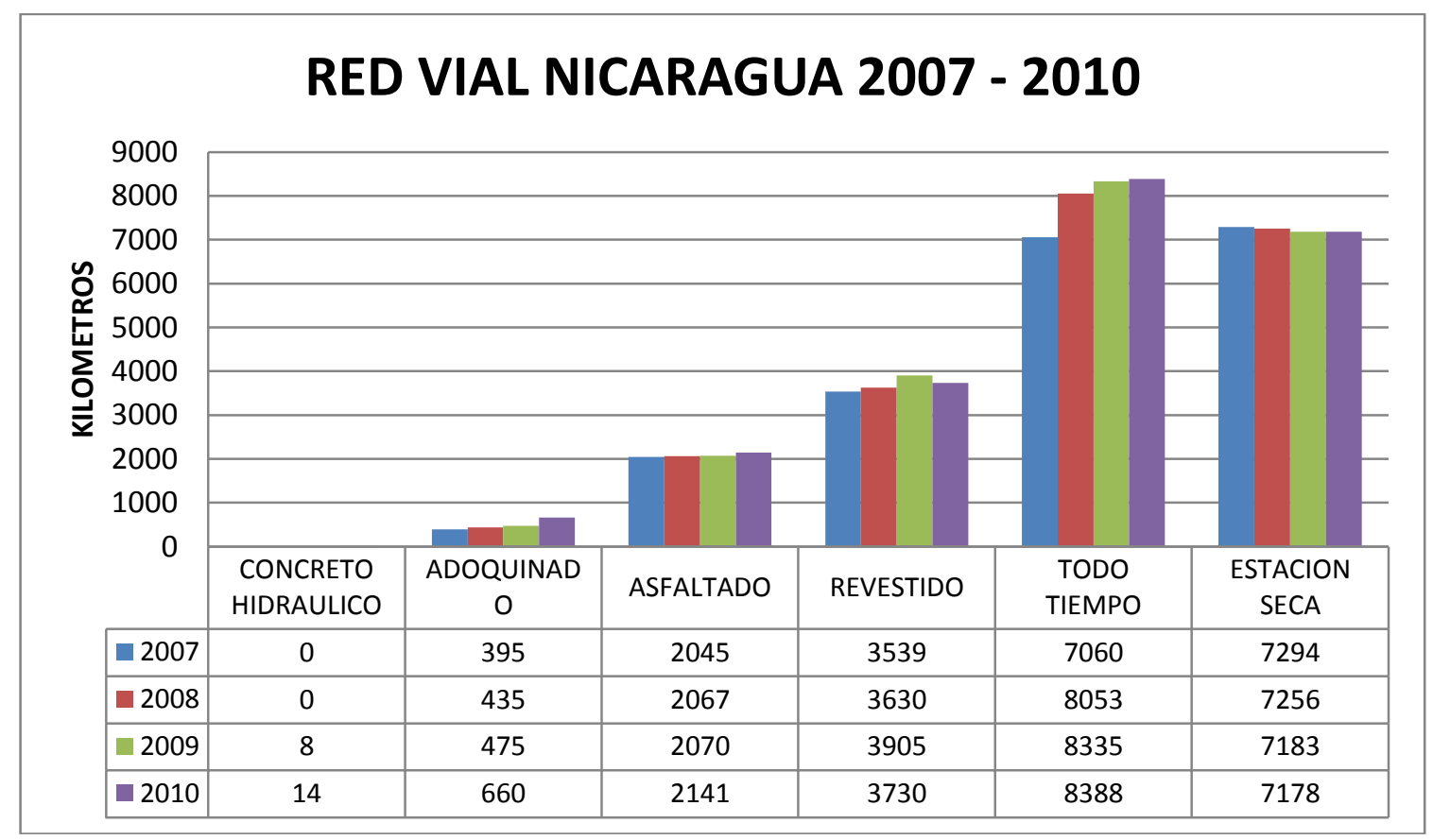

Fig. 1 Red Vial Nicaragua 2007-2010

(Elaboración con base a datos sistematizados de la División General de Planificación-MTI) 
Tabla 4. Costos unitarios de construcción para pavimentos flexibles

\begin{tabular}{|c|c|c|}
\hline Estructura & UM & $\begin{array}{c}\text { Costo Unitario } \\
\text { USD }\end{array}$ \\
\hline Carpeta de Concreto Asfáltico & $\mathrm{M}^{3}$ & 230 \\
\hline $\begin{array}{c}\text { Asfalto Rebajado RC-250 para } \\
\text { riego de liga }\end{array}$ & $\mathrm{M}^{2}$ & 0,44 \\
\hline Material de Secado & $\mathrm{M}^{2}$ & 0,19 \\
\hline Asfalto MC-70 para imprimación & $\mathrm{M}^{2}$ & 0,84 \\
\hline $\begin{array}{c}\text { Base estabilizada con cemento } \\
\text { (4\%) }\end{array}$ & $\mathrm{M}^{3}$ & 40,29 \\
\hline Base Triturada & $\mathrm{M}^{3}$ & 27,42 \\
\hline Sub-base granular & $\mathrm{M}^{3}$ & 25,19 \\
\hline
\end{tabular}

Fuente: Ventajas de los Pavimentos de Concreto Hidráulico. Díaz, Ricardo. INCYC, Managua, Nicaragua.

Tabla 5. Costos unitarios de construcción para pavimentos rígidos

\begin{tabular}{|c|c|c|}
\hline Estructura & $\mathbf{U M}$ & $\begin{array}{c}\text { Costo Unitario } \\
\text { USD }\end{array}$ \\
\hline $\begin{array}{c}\text { Concreto Hidráulico (MR=50 } \\
\mathrm{kg} / \mathrm{cm}^{2}\end{array}$ & $\mathrm{M}^{3}$ & 185 \\
\hline Sello de Juntas & $\mathrm{ml}$ & 0,25 \\
\hline Canastilla de Dovelas & $\mathrm{ml}$ & 1,50 \\
\hline $\begin{array}{c}\text { Base Estabilizada con } \\
\text { Cemento (3.5\%) }\end{array}$ & $\mathrm{M}^{3}$ & 35 \\
\hline
\end{tabular}

Este precio incluye: suministro, colocación, curado y corte.

Fuente: Ventajas de los Pavimentos de Concreto Hidráulico. Díaz, Ricardo. INCYC, Managua, Nicaragua.

\section{Ventajas de los pavimentos de concreto hidráulico}

Desde su introducción a la construcción de carreteras en Inverness, Escocia (Pereira, 1977), los pavimentos de concreto hidráulico han demostrado su innegable capacidad para soportar el tránsito durante prolongados espacios de tiempo, alcanzando periodos de vida útil de hasta 50 años o más, aun cuando el periodo de diseño es considerablemente menor.

Más allá de su durabilidad y resistencia, los pavimentos de concreto hidráulico presentan un gran número de ventajas en comparación con otros tipos de pavimentos. Estas abarcan diferentes ámbitos de interés para toda la sociedad, desde la economía (tanto de las oficinas de administración vial, como de los usuarios), la seguridad vial, el ahorro energético, el desarrollo sostenible (equilibrio entre las exigencias medioambientales, las necesidades sociales y las consideraciones económicas), la disminución de la contaminación (presente en la producción del cemento y a lo largo de la producción del concreto), la innovación tecnológica, entre otros factores. (Tabla 5. Costos Unitarios de Construcción para Pavimentos Rígidos).

Tabla 6. Calendario de intervenciones para mantenimiento de pavimento flexible sobre base granular

\begin{tabular}{|c|c|c|c|}
\hline $\begin{array}{c}\text { Intervención } \\
\text { (Año) }\end{array}$ & Actividad & Unid. & Cantidad \\
\hline \multirow{2}{*}{4} & $\begin{array}{l}\text { Bacheo } \\
\text { Superficial }\end{array}$ & $\mathrm{M}^{3}$ & $3-5$ \\
\hline & $\begin{array}{l}\text { Sello de Grietas } \\
\text { Mecanizado }\end{array}$ & $\mathrm{M}^{2}$ & $\begin{array}{l}6,570- \\
7,300 \\
\end{array}$ \\
\hline \multirow{3}{*}{7} & $\begin{array}{l}\text { Bacheo } \\
\text { Superficial }\end{array}$ & $M^{3}$ & $6-10$ \\
\hline & $\begin{array}{l}\text { Bacheo } \\
\text { Profundo }\end{array}$ & $\mathrm{M}^{3}$ & $\begin{array}{l}11.25- \\
15.00\end{array}$ \\
\hline & $\begin{array}{l}\text { Tratamiento } \\
\text { Superficial }\end{array}$ & $\mathrm{M}^{2}$ & $\begin{array}{l}6,570- \\
7,300 \\
\end{array}$ \\
\hline \multirow{3}{*}{10} & $\begin{array}{ll}\text { Recarpeteo de } \\
\text { Refuerzo }\end{array}$ & $\mathrm{M}^{3}$ & $197-365$ \\
\hline & $\begin{array}{l}\text { Bacheo } \\
\text { Superficial }\end{array}$ & $\mathrm{M}^{3}$ & $6-10$ \\
\hline & $\begin{array}{l}\text { Bacheo } \\
\text { Profundo }\end{array}$ & $\mathbf{M}^{3}$ & $15-20$ \\
\hline \multirow{2}{*}{13} & $\begin{array}{l}\text { Bacheo } \\
\text { Superficial }\end{array}$ & $\mathrm{M}^{3}$ & $4-7$ \\
\hline & $\begin{array}{l}\text { Sello de Grietas } \\
\text { Mecanizado }\end{array}$ & $\mathrm{M}^{2}$ & $\begin{array}{l}6,570- \\
7,300 \\
\end{array}$ \\
\hline \multirow{3}{*}{16} & $\begin{array}{l}\text { Bacheo } \\
\text { Superficial }\end{array}$ & $\mathrm{M}^{3}$ & $9-15$ \\
\hline & $\begin{array}{l}\text { Bacheo } \\
\text { Profundo }\end{array}$ & $\mathrm{M}^{3}$ & $15-20$ \\
\hline & $\begin{array}{l}\text { Tratamiento } \\
\text { Superficial }\end{array}$ & $\mathrm{M}^{2}$ & $\begin{array}{l}6,570- \\
7,300 \\
\end{array}$ \\
\hline
\end{tabular}

Fuente: ICC Consultores e INCYC, Nicaragua

¿Qué pasa con los costos en el tiempo?

Los costos del proyecto aumentan. En promedio, un pavimento asfáltico puede llegar a costar hasta tres veces más que su equivalente pavimento de concreto. El aumento en los precios del crudo, incrementa los costos y los hace más impredecibles. (Tabla 4. Costos Unitarios de Construcción para Pavimentos Flexibles).

Además de ello, cabe mencionar, que la diferencia en el costo del Ciclo de Vida entre pavimentos asfálticos y pavimentos de concreto hidráulico, sería más 
significativa si se tomase en cuenta el costo de los usuarios (especialmente el gasto de combustible).

\section{Tabla 7. Avance Físico (en Km)}

\begin{tabular}{|c|c|}
\hline Meses & Cantidad \\
\hline Enero & 621.36 \\
\hline Febrero & 99.00 \\
\hline Marzo & 185.13 \\
\hline Abril & 124.12 \\
\hline Mayo & 126.93 \\
\hline Junio & 111.58 \\
\hline Julio & 130.58 \\
\hline Agosto & 97.89 \\
\hline Septiembre & 37.57 \\
\hline Octubre & 38.46 \\
\hline Noviembre & \\
\hline Diciembre & \\
\hline Total & 1572.62 \\
\hline \% Avance & 102.19 \\
\hline
\end{tabular}

Tabla 8. Avance financiero (millones de C\$)

\begin{tabular}{|c|c|}
\hline Meses & Desembolsos \\
\hline Enero & 157.96 \\
\hline Febrero & 179.13 \\
\hline Marzo & 475.11 \\
\hline Abril & 173.26 \\
\hline Mayo & 234.73 \\
\hline Junio & 105.00 \\
\hline Julio & 135.49 \\
\hline Agosto & 128.83 \\
\hline Septiembre & 52.36 \\
\hline Octubre & 305.28 \\
\hline Noviembre & \\
\hline Diciembre & \\
\hline Total & 1947.16 \\
\hline \% Avance & 72.54 \\
\hline
\end{tabular}

Fuente de información: SIGFA al 31 de octubre del 2012 División de Control Técnico (Seguimiento PIP)

\section{CONCLUSIONES}

En la búsqueda de las mejores soluciones para pavimentos de carretera, no puede obviarse la utilización de soluciones técnicas que puedan ofrecer beneficios económicos, en términos de ahorros netos (inversión y mantenimiento en el largo plazo). Hay múltiples estudios económicos que comprueban que transitar en concreto, es más económico que el asfalto. El asfalto se deforma, y al hacerlo, consume energía. Al consumir más energía, hay más gases contaminantes. Este es un buen punto para las agencias viales (Ministerio de Transporte e Infraestructura-MTI, Fondo de Mantenimiento Vial-FOMAV y alcaldías municipales), en el caso de Nicaragua.

Con el concreto hidráulico se contamina menos y es más durable, si bien es cierto que su inversión inicial es mayor. Además de ello, el mantenimiento es mínimo, drena mejor el agua y presenta mayor resistencia a sucumbir, producto de las escorrentías superficiales. Los espesores del concreto pueden variar, pero dependen principalmente del volumen del tráfico proyectado y de las características de la flota vehicular a circular.

Por otro lado, el asfalto tiene un deterioro permanente, porque es una degeneración propia del material: mantenimiento cada dos o cuatro años; recarpeteos mayores, cada ocho años; reconstrucción total, cada doce años. En cambio, el concreto hidráulico puede tener el primer mantenimiento a los diez años. (Tabla 6. Calendario de Intervenciones para Mantenimiento de Pavimento Flexible sobre Base Granular).

En todos los casos, la inversión inicial del pavimento flexible sobre base estabilizada con cemento, resulta màs económica que cualquier otra alternativa; siendo hasta casi un $30 \%$ màs económico que el pavimento flexible con base granular.

El costo de mantenimiento para el pavimento de concreto asfâltico, resulta de 10 a 15 veces mayor, que el del pavimento de concreto hidraùlico. Estos resultados concuerdan con los diferentes resultados obtenidos de las diferentes bibliografías consultadas.

Se pudo determinar, para todos los casos analizados y en base a estudios de especialistas en la materia, que la opción que optimiza los recursos a lo largo de su ciclo de vida, es la estructura de pavimento con concreto hidráulico. Resultando en comparación con el pavimento flexible sobre base granular un 20\% más económico para losas convencionales y hasta un $34 \%$ para losas cortas.

Otro punto muy importante, es que no se debe de construir solo por hacerlo, aparte de la justificación económica y social del proyecto, la solución tomada debe sustentarse y ajustarse a las necesidades dentro del periodo de diseño; es decir, que la solución debe ser sostenible en el tiempo, con argumentos técnicoseconómicos congruentes y concienzudamente elaborados.

Además, consideramos que es necesario:

-Mejorar el sistema de información de conteos volumétricos, pesos y dimensiones, que abarque toda la 
red vial, y, que actualmente es parcial. Los mismos se iniciaron en 1954, llevándose registros de aforo de tráfico hasta 1979. Se interrumpieron en el período de 1980 a 1995, por guerra civil y otros, y fue hasta el año de 1996 que la empresa CARL BRO GROUP de procedencia danesa, hizo la implementación del

Sistema de Administración de Pavimentos, en donde mejoró los registros y reactivó el sistema de conteos de tráfico.

-El Ministerio de Transporte e Infraestructura (MTI), tiene como objetivo principal en el área de estudio de tráfico, realizar conteos y clasificación de vehículos en toda la Red Vial Nacional, permitiendo con esto medir la demanda de transporte del sistema de carreteras.

- Debe de existir e implementarse una relación entre el diseño de capacidades de carga y el volumen de tráfico, para poder establecer un diseño real de estructuras de pavimentos y caminos, soportados en estudios de mecánica de suelos y afines; para poder obtener una relación integral y evitar de esa manera, el desperdicio de recursos humanos y financieros, tan escasos y necesarios, ante las necesidades de mantenimiento que requiere de forma continua y planificada la red vial.

-Para apreciar el efecto de daño causado por los distintos tipos de vehículos que circulan en la Red Vial, podemos hacer las siguientes observaciones: a) La relación entre el peso y el daño provocado sobre un pavimento no es lineal, sino exponencial; b) Los camiones y autobuses, son los responsables de la mayoría de los daños en los pavimentos; c) en el diseño de pavimentos un elemento básico y fundamental es la estimación de la carga de tràfico en términos de ESAL, que un pavimento deberá soportar en su vida de diseño.

-Las experiencias desarrolladas en el país, en la rehabilitación de pavimentos, durante los últimos quince años, deben ser tomadas en debida cuenta, en el diseño de los pavimentos

El incremento desmesurado de los precios del asfalto y sus derivados, y los niveles de tráfico (principalmente pesados) actuales que definen las tendencias del tràfico proyectado, principalmente para las carreteras de mayor volumen; hacen que el dilema de los ingenieros de pavimento y de las agencias a cargo de estos vuelve a ser: ¿cuál es la alternativa óptima que combine confort, durabilidad y beneficio económico?. Tradicionalmente se ha aceptado que el costo de inversión (concreto con cemento Portland), es superior al costo de los pavimentos flexibles.
-La ventaja de los pavimentos rígidos se ha puesto de manifiesto en el largo plazo, debido a los bajos costos de mantenimiento en el periodo de diseño del proyecto. Dichos criterios, en conjunto con los actuales precios del petróleo y los correspondientes precios que ha llegado a alcanzar la producción y colocación de mezcla asfáltica, sustentan la necesidad de realizar en cada proyecto un Análisis en el Ciclo de Vida del Proyecto (Cycle Cost Analysis), como lo recomienda la propia guía AASHTO.

-Es necesario mencionar que este análisis (ACCV)(Análisis del Costo del Ciclo de Vida) es una herramienta financiera, y en ningún momento predice el desempeño de la vía. La forma más común del ACCV es mediante el cálculo del Valor Presente Neto (VPN), que es la suma de todos los costos y beneficios del proyecto, en términos monetarios. Utiliza costos iniciales con costos futuros de mantenimiento. Estos costos se deducen utilizando una tasa de descuento adecuada al valor en el tiempo del dinero.

-En las actas de recepción y entrega de obras, se debe de adjuntar siempre el plan de mantenimiento respectivo, al cual no se le da el seguimiento técnico requerido por la instancia correspondiente. De esta forma, al no existir planificación, seguimiento y control; se reduce la vida útil de la red vial de Nicaragua.

-Se tiene que tener a disposición un inventario documental de la red vial, que registre desde el diseño de la misma, datos técnicos de la ejecución de obras, los mantenimientos preventivos planificados y correctivos, de que la misma ha sido objeto durante su vida útil y posterior recuperación. Este es un

trabajo que fortalecería la planificación de la gestión institucional, el cumplimiento de normas, especificaciones técnicas y recursos asignados.

-Es necesario, proponer a las autoridades gubernamentales, que poco a poco se vaya cambiando la Matriz Vial, de asfalto o adoquín, a concreto hidráulico; porque así las ventajas competitivas para el país, se incrementarían; dado que uno de los pilares fundamentales para el desarrollo de un país, es su infraestructura vial. Todo esto tomando en consideración los tipos de clasificaciones usadas en Nicaragua. (Wilbur Smith and Associates y Cisneros y Conrado, Co. Ltda. 1976. Plan Nacional de Transporte). 
-La recesión económica mundial obliga a países dependientes como el nuestro, a implementar y tomar medidas, entre otras, adoptando prácticas de eficiencia y calidad en función de los recursos asignados. Para ello el MTI debe cumplir en lo posible con las especificaciones técnicas y normas de diseño acordes con las exigencias actuales del tráfico nacional, lo que viene a garantizar en gran medida la rentabilidad en la inversión de la infraestructura.

Esperamos que éste artículo, les sirva a los interesados de estímulo en su esfuerzo profesional, y para, enfrentar las dificultades que implica investigar, en un país, con ciencia incipiente y carente de recursos.

\section{REFERENCIAS}

Archondo-Callao, Rodrigo. (1995 $)$. HDM Manager Version 3.0: User-friendly Shell Environment for the Highway Design and Manintenance Standard Model (HDM). Methods for Economic Evaluation of Highways Investment and Maintenance. Transport Division, Transportation, Water \& Urban Development Department, the World Bank. Washington D.C.: The World Bank.

CORASCO-PAST DANIDA, MTI. (2008). Manual para la elaboración de Especificaciones Técnicas.

Díaz, Ricardo (Setp. 2010). Ventajas de los Pavimentos de Concreto Hidráulico. INCYC, Managua, Nicaragua.

FOMAV, (2008-10). Memoria de Labores.

JICA, (1994). Estudio sobre Mejoramiento y Rehabilitación de Carreteras en la República de Nicaragua: Informe Final, volumen I, Plan Maestro. Elaborado por Central Consultant Inc., Nippon Koei Co., Ltd. Para la Agencia de Cooperación Internacional del Japón (JICA).
Ingenieros Consultores Centroamericanos, Managua (s.n.). Soluciones de Pavimentos en Nicaragua: Comparación técnica y económica.

MTI, (2010). Memoria Institucional.

MTI, (2006). Plan de Conservación Vial. Dirección General de Vialidad.

Pereira, Juan E. (1977). La historia del cemento, el concreto y el concreto reforzado. Publicación periódica, s.I. Civil Engineering.

Red Vial, (2008). Oficina de Inventario Vial. División de Administración Vial. División General de Planificación. Ministerio de Transporte e Infraestructura MTI. Nicaragua.

Wilbur Smith and Associates y Cisneros y Conrado Co. Ltda. (1976). Plan Nacional de Transporte.

Zaniewski, John P. (1989). Effect of Pavement Surface Type on Fuel Consumption. Portland Cement Association.

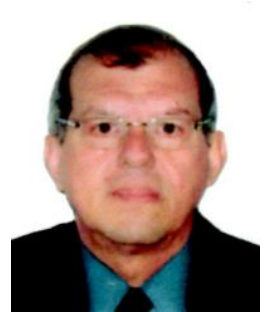

Wilfredo Martínez se graduó de Ingeniero Civil, en la Universidad Nacional de Ingeniería (UNI) en 1994. Es también graduado en postgrados en: Formulación de Proyectos de Inversión y Finanzas (1989), así como, el grado de Maestría en Administración Funcional (UNANManagua-2000). Además, es Ingeniero Electromecánico (1983) e Ingeniero Agrícola (1987). Actualmente es Doctorante en Ciencias Económicas, en la UNANManagua y LUZ de Venezuela. 